\title{
Effect of omeprazole on antihypertensive efficacy of amlodipine in patients with comorbid pathology - arterial hypertension and acid-dependent disease
}

\author{
Margarita N. Dorofeeva ${ }^{1}$, Evgeniya V. Shikh¹, Zhanna M. Sizova' ${ }^{1}$, Natalya N. Shindryaevaa ${ }^{1}$ Natalya I. Lapidus ${ }^{1}$
}

\begin{abstract}
Objective: The problem of drug interactions is increasingly important today because they may induce serious adverse events as well as interfere with efficacy of pharmacotherapy. Combinations of drugs are most often prescribed to patients presenting with comorbid pathology. The incidence of a combination of arterial hypertension (AH) and acid-dependent diseases (ADDs) varies widely ranging from 11.6 to $50 \%$. One of combinations of drugs prescribed to such patients is a combination of calcium channel blocker amlodipine and proton pump inhibitor omeprazole. The latter in the human body undergoes biotransformation mediated at the level of cytochrome P450 by isoenzymes CYP2C19 and CYP3A4. Amlodipine is a substrate of the isoenzyme CYP3A4, which increases the probability of the development of interaction between these drugs. The purpose of our study was to investigate antihypertensive efficacy of amlodipine in patients suffering from arterial hypertension combined with acid-dependent diseases and additionally taking omeprazole.

Method: Study included a total of 150 patients with AH and ADD. Antihypertensive therapy was evaluated by means of office measuring of arterial pressure (AP) and circadian monitoring of AP (CMAP). The followed-up patients with AH and ADD were divided into 2 groups. Group One was composed of hypertensive patients undergoing pharmacotherapy with $10 \mathrm{mg}$ amlodipine, whose condition required due to exacerbation of ADD administration of omeprazole at a dose of $20 \mathrm{mg}$ for a period from 3 to 4 weeks. Group Two comprised hypertensive patients receiving antihypertensive therapy consisting of $10 \mathrm{mg}$ amlodipine, who were found to have remission of acid-dependent diseases, with no additional medication taken.

Results: The obtained findings demonstrated that one of the commonly used drug combinations in treatment of patients with AH and ADD in ambulatory conditions was a combination of omeprazole and amlodipine, accounting for $7.1 \%$. The results of office measurement of arterial pressure (AP) 2 weeks after initiating pharmacotherapy with omeprazole in patients with AH and ADD demonstrated that the patients receiving omeprazole in addition to antihypertensive therapy were found to have a statistically significant decrease in systolic arterial pressure (SAP) and statistically significantly more pronounced dynamics of a decrease in diastolic arterial pressure (SAP) $(p<0.05)$ compared with those not receiving therapy with omeprazole. Also, in the group of patients taking omeprazole, the findings of circadian monitoring of blood pressure (CMAP) showed a statistically significant decrease in average circadian SAP, average circadian DAP, mean value of daytime SAP and mean value of nighttime SAP $(p<0.05)$.

Conclusion: The obtained findings demonstrated that simultaneous prescription of amlodipine and omeprazole to patients with concomitant pathology, i.e., $A H$ and $A D D$, turned out to enhance the antihypertensive affect of amlodipine, which probably resulted from substrate competition of amlodipine and omeprazole at the level isoenzyme CYP 3A4 of cytochrome P450.
\end{abstract}

Keywords: omeprazole, amlodipine, isoenzyme CYP 3A4, arterial hypertension, acid-dependent diseases

\section{INTRODUCTION}

Drug interaction is currently an increasingly important problem of modern pharmacotherapy since it may considerably alter the pharmacodynamic activity of drugs and be a cause of failed therapy or a decrease of its safety (14).

Widely used for treatment of acid-dependent diseases, omeprazole in the human body undergoes rapid and intensive biotrasformation mediated by isoenzymes CYP2C19 and CYP3A4, thus increasing the probability of the development of drug interaction with other substrates or inhibitors of these enzymes $(5,6)$. The question arises of whether this alteration in the activity of isoenzyme CYP3A4 can lead to clinically significant interactions (altered activity) of drugs metabolised

\footnotetext{
1 Chair of medical and social assessment, urgent and ambulatory care. First Moscow State Medical University named after I. M. Sechenov, Moscow, Russia
}

Correspondence: Margarita N. Dorofeeva

Chair of, of medical and social assessment, urgent and ambulatory care

First Moscow State Medical University named after I. M. Sechenov, Moscow, Russia

Received: 9 Jan 2018, Accepted: 13 Mar 2018

E-mail: margaritadorofeeva@yandex.com

(C) 2018 by the authors; licensee Modestum Ltd., UK. This article is an open access article distributed under the terms and conditions of the Creative Commons Attribution License (http://creativecommons.org/licenses/by/4.0/).

Electronic Journal of General Medicine 
Table 1: Clinical characteristics of patients with $A H$ and $A D D$ enrolled into the study

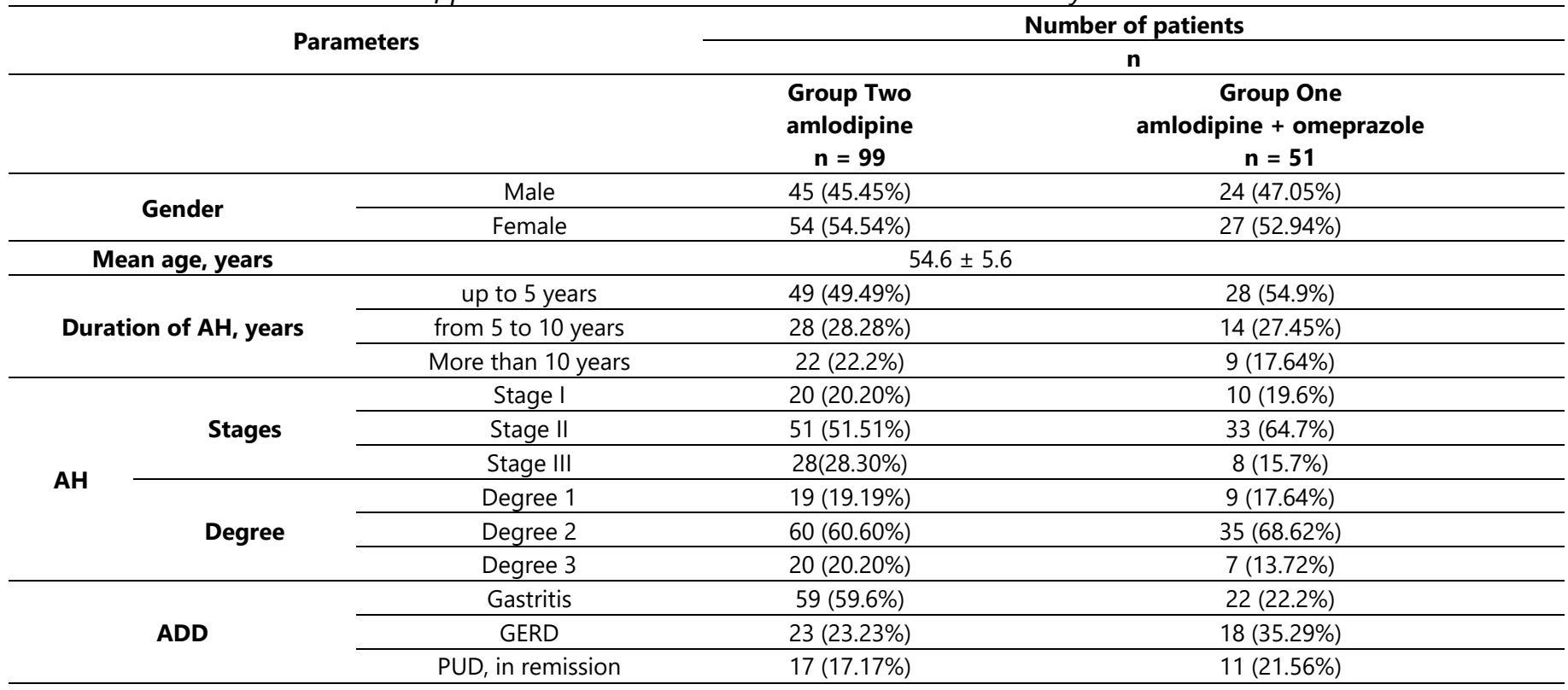

via this enzyme and influence the clinical efficacy of drugs $(7,8)$. Taking into consideration frequent administration of omeprazole in patients with $\mathrm{ADD}$ and $\mathrm{AH}$ in ambulatory practice, this problem requires further study.

\section{MATERIALS AND METHODS}

At the first stage of the study we analysed a total of 1,126 out-patient medical case histories in order to reveal the incidence rate of the combination of $A H$ and ADD in the setting of a municipal polyclinic.

At the second stage of the study we analysed pharmacotherapy of patients with accompanying pathology - ADD (analysis of the most commonly prescribed combinations of drugs, drug interactions with regard for metabolism, as well as assessment of the risk for the development of adverse drug reactions (ADRs) at the level of cytochrome P450 CYP3A4 with the help the Internet-resource drugs.com).

At the third stage, the study included a total of 150 patients with AH and ADD, aged above 18 years, followed up in the ambulatory-polyclinic link and having signed informed consent for participating in the study. The exclusion criteria were as follows: a history of endured acute impairment of cerebral circulation and transitory ischaemic attack, myocardial infarction during the previous year, severe cardiac arrhythmia and impaired conductivity, chronic heart failure, chronic liver diseases, chronic kidney disease, adrenal diseases, and patients taking glucocorticoids.

To be included into the study, all patients underwent a standard procedure: taking case history, anthropometric measurements (height, weight), physical examination, instrumental and laboratory examination.

Antihypertensive therapy was evaluated by means of two methods: office measuring of AP and circadian monitoring of AP (CMAP). Office measurement of AP was carried out at each visit, CMAP was conducted two times: before starting to take a combination of amlodipine with omeprazole and at 2 weeks of combined pharmacotherapy.

Office measurement of AP was performed in a seated position after a 15-minute rest period by the Korotkov technique, measuring AP twice with a 3-minute interval, followed by calculation of the mean value. Circadian monitoring was carried out during 24 hours by means of the Russian-made software-hardware complex "BPLab".

The followed-up patients with AH and ADD were divided into 2 groups. Group One was composed of hypertensive patients undergoing pharmacotherapy with $10 \mathrm{mg}$ amlodipine, whose condition required due to exacerbation of ADD administration of omeprazole at a dose of $20 \mathrm{mg}$ for a period from 3 to 4 weeks.

Group Two comprised hypertensive patients receiving antihypertensive therapy consisting of $10 \mathrm{mg}$ amlodipine, who were found to have remission of acid-dependent diseases, with no additional medication taken (Table 1).

\section{RESULTS}

The analysis of 1,126 ambulatory medical case histories of patients with AH followed up at the Municipal Polyclinic № 2 of the Moscow Healthcare Department showed that 150 (13\%) patients with AH were diagnosed with ADD. This 
cohort was represented predominantly by 30 -to-67-year-old patients with chronic gastritis (58\%). The clinical characteristics of these patients are shown in Table 1.

Analysing the names and frequency of prescribing antihypertensive drugs demonstrated that most frequently prescribed were the following ACE inhibitors: perindopril - 23 (4.4\%) patients, enalapril - 30 (6.2\%), lisinopril - 17 (3.5\%), fosinopril - 7 (1.5\%), berliprll - $2(0.4 \%)$, quinapril - $1(0.2 \%)$ patient. As angiotensin II receptor antagonist (ARA II) the following drugs were used: valsartan in 21 (4.4\%) patients, lozartran in 32 (6.6\%) patients, olmesartan in 11 (2.3\%) patients and azilsartan in $8(1.7 \%)$. Amongst beta-adrenoblockers (BABs) most often used were the following drugs: bisoprolol in $41(8.5 \%)$ patients, metoprolol $-25(5.3 \%)$ subjects, atenolol in $5(1.1 \%)$ patients, carverdiol in $3(0.6 \%)$ patients, and betaxolol in 13 (2.7\%) patients. Of the calcium channel blockers, amlodipine was taken by 42 (8.7\%) patients, felodipine by 19 (3.9\%) patients, lercanidipine by 8 (1.7\%). Of diuretics, mention should be made of indapamide $-9(1.9 \%)$ patients, and hydrochlorothiazide -18 (3.7\%) patients.

Hence, the most often used drugs possessing antihypertensive activity in patients with AH and ADD in ambulatory practice were bisoprolol taken by $8.5 \%$ and amlodipine taken by $8.7 \%$.

Analysing the names and frequency of prescribing antisecretory drugs demonstrated that patients with concomitant pathology, i.e. AH and ADD, most often received proton pump inhibitors: omeprazole - 92 (19.1\%) patients, pantoprazole - 27 (5.6\%), and esomeprazole - 19 (3.9\%) patients. Of another group of agents decreasing secretion of hydrochloric acid mention should be made of histamine $\mathrm{H}_{2}$-receptor blockers such as ranitidine $-3(0.6 \%)$ cases and famotidine -9 (1.9\%) cases.

Analysing combined pharmacotherapy in 150 patients with $\mathrm{AH}$ and ADD demonstrated that amongst a total of 92 cases of omeprazole with ACE inhibitors, most often encountered were combinations with enalapril - 18 (3.9\%) patients, perindopril - 11 (2.4\%) patients, lisinopril - 7 (1.5\%) patients and fosinopril - 7 (1.5\%) patients.

Amongst combinations of omeprazole with ARAs II most often encountered was omeprazole combined with valsartan - 20 (4.3\%) patients. Omeprazole combined with losartan was prescribed to 8 (1.7\%) patients and with olmesartan to 5 (1.1\%) patients.

Amongst combinations of omeprazole with $B A B s$, most frequently encountered was a combination with bisoprolol 28 (6.1\%) patients, metoprolol - 19 (4.1\%). A combination of omeprazole and carverdiol was prescribed in $0.6 \%$ of cases.

Of the combinations of omeprazole with calcium channel blockers, most often encountered was a combination with amlodipine -33 (7.1\%) cases. Simultaneous administration of omeprazole and felodipine accounted for $1.9 \%$ (9 patients).

Pantoprazole was prescribed in the composition of 19 various combinations with antihypertensive drugs, amongst which mention can be made of the most frequently used in out-patient practice combinations: with enalapril - $6(1.3 \%)$ patients, valsartan $-4(0.9 \%)$, losartan $-6(1.3 \%)$, and bisoprolol $-6(1.3 \%)$ patients.

Esomeprazole was prescribed in the composition of 12 various combinations with antihypertensive drugs: most often with perindopril $-6(1.3 \%)$ patients, valsartan $-3(0.6 \%)$, amlodipine $-4(0.9 \%)$, metoprolol $-5(1.1 \%)$, bisoprolol -4 (0.9\%) patients (Table 1).

Hence, the most commonly used in out-patient practice combinations in treatment of patients with AH and ADD were as follows: omeprazole with enalapril - 18 (3.9\%) patients, with valsartan - 20 (4.3\%), with bisoprolol - 28 (6.1\%), with metoprolol -19 (4.1\%). The most frequently used combination in treatment of patients with AH and ADD according to the findings of the analysis of medical case histories was a combination of omeprazole and amlodipine: 33 (7.1\%) patients (Figure 1).

We examined the dynamics of $A P$ in patients with $A H$ and $A D D$, being on adjusted antihypertensive therapy consisting of amlodipine at a dose of $10 \mathrm{mg}$, who due to exacerbation of ADD were additionally prescribed omeprazole at a dose of $20 \mathrm{mg}$.

The results of office measurement of AP 2 weeks after the beginning of pharmacotherapy with omeprazole in patients with $\mathrm{AH}$ and $\mathrm{ADD}$ demonstrated that the patients receiving antihypertensive therapy with amlodipine supplemented by omeprazole due to exacerbation of ADD were found to have a statistically significant decrease in SAP, statistically significantly more pronounced dynamics of a decrease in DAP $(p<0.05)$ as compared with the group of patients not receiving therapy with omeprazole. 


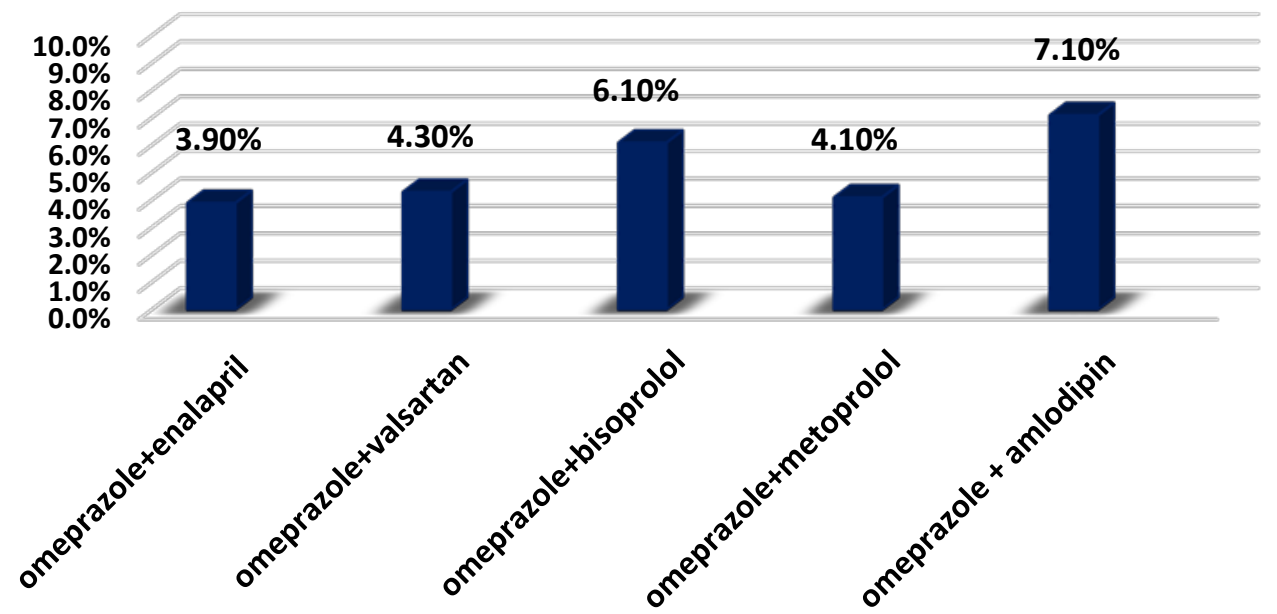

Figure 1: Most frequently prescribed in out-patient practice combinations of drugs with antihypertensive and antisecretory activity

Table 2: Dynamics of AP in patients on the background of pharmacotherapy

\begin{tabular}{|c|c|c|c|}
\hline \multicolumn{4}{|c|}{ 24-hour monitoring of AP } \\
\hline \multirow{2}{*}{ Mean circadian SAP, mm Hg } & $137.3 \pm 4.8$ & $135.7 \pm 3.9$ & \\
\hline & $132.5 \pm 5.5$ & $123.5 \pm 4.3$ & 0.04 \\
\hline Dynamics of mean office SAP & $5.1 \pm 1.5$ & $12.7 \pm 2.7$ & 0.04 \\
\hline Dynamics of mean office SAP in $\Delta \%$ & $3.7 \pm 0.8$ & $10.3 \pm 3.4$ & 0.04 \\
\hline In-group p & 0.04 & 0.03 & \\
\hline \multirow{2}{*}{ Mean DAP circ., mm Hg } & $86.7 \pm 3.4$ & $86.7 \pm 3.1$ & \\
\hline & $82.4 \pm 3.4$ & $75.5 \pm 3.9$ & 0.06 \\
\hline Dynamics of average circadian DAP & $4.7 \pm 1.7$ & $11.2 \pm 2.9$ & 0.04 \\
\hline Dynamics of average circadian DAP in $\Delta \%$ & $4.9 \pm 1.6$ & $12.91 \pm 3.5$ & 0.04 \\
\hline In-group p & 0.04 & 0.04 & \\
\hline \multirow{2}{*}{ Mean daytime SAP, mm Hg } & $137.6 \pm 4.6$ & $136.9 \pm 4.4$ & \\
\hline & $135.9 \pm 5.1$ & $122.6 \pm 5.7$ & 0.04 \\
\hline Dynamics of mean daytime SAP, $\mathrm{mm} \mathrm{Hg}$ & $1.7 \pm 0.7$ & $14.3 \pm 2.6$ & 0.04 \\
\hline Dynamics of mean daytime SAP, $\mathrm{mm} \mathrm{Hg} \Delta \%$ & $1.23 \pm 2.2$ & $10.4 \pm 2.8$ & 0.04 \\
\hline In-group p & 0.04 & 0.04 & \\
\hline \multirow{2}{*}{ Mean nighttime SAP, mm Hg } & $131.2 \pm 4.3$ & $130.5 \pm 4.27$ & \\
\hline & $128.5 \pm 5.2$ & $120.4 \pm 4.8$ & 0.04 \\
\hline Dynamics of mean nighttime SAP, $\mathrm{mm} \mathrm{Hg}$ & $3.61 \pm 1.1$ & $9.9 \pm 1.8$ & 0.04 \\
\hline Dynamics of mean nighttime SAP, $\mathrm{mm} \mathrm{Hg} \Delta \%$ & $2.75 \pm 1.3$ & $7.5 \pm 2.1$ & 0.04 \\
\hline In-group p & 0.04 & 0.04 & \\
\hline \multirow{2}{*}{ Mean daytime DAP, mm Hg } & $87.3 \pm 3.5$ & $87.5 \pm 3.7$ & \\
\hline & $83.6 \pm 5.7$ & $80.7 \pm 5.6$ & 0.06 \\
\hline Dynamics of mean daytime DAP, $\mathrm{mm} \mathrm{Hg}$ & $4.38 \pm 1.3$ & $6.8 \pm 2.2$ & 0.06 \\
\hline Dynamics of mean daytime $\mathrm{DAP}, \mathrm{mm} \mathrm{Hg} \Delta \%$ & $5.2 \pm 1.8$ & $7.9 \pm 2.1$ & 0.06 \\
\hline In-group p & 0.04 & 0.02 & \\
\hline \multirow{2}{*}{ Mean nighttime DAP, mm Hg } & $85.8 \pm 6.1$ & $85.7 \pm 6.2$ & \\
\hline & $76.8 \pm 5.9$ & $74.5 \pm 6.3$ & 0.05 \\
\hline Dynamics of mean nighttime $\mathrm{DAP}, \mathrm{mm} \mathrm{Hg}$ & $9.2 \pm 2.1$ & $11.2 \pm 2.3$ & 0.05 \\
\hline Dynamics of mean nighttime DAP, $\mathrm{mm} \mathrm{Hg} \Delta \%$ & $10.48 \pm 3.4$ & $13.06 \pm 2.7$ & 0.05 \\
\hline
\end{tabular}

The results of CMAP 2 weeks after the beginning of pharmacotherapy with omeprazole in patients with AH and ADD demonstrated that the patients additionally to therapy taking omeprazole had a statistically significant decrease in average circadian SAP, average circadian DAP, average value of daytime SAP, mean value of nighttime SAP ( $p<0.05$ ) as compared with the group of patients not receiving therapy with omeprazole. The patients undergoing therapy supplemented with omeprazole were also found to have a slightly more pronounced decrease in mean daytime DAP and nighttime DAP, which, however, was not statistically significant ( $p>0.05)$ (Table 2). 


\section{DISCUSSION AND CONCLUSIONS}

The findings of analyzing the primary medical records of patients demonstrated that the frequency of combination of $\mathrm{AH}$ and $\mathrm{ADD}$ in out-patient clinical practice amounted to $13 \%$.

The incidence and probability of concomitant lesions of the digestive and cardiovascular systems are determined by the commonness of many factors responsible for the development of both gastroenterological and cardiovascular diseases, in particular, an increase in the aggregation properties of erythrocytes and blood platelets, systemic impairments at the level of arterioles, hypertrophy of smooth-muscle cells of vessels, impaired endothelium-derived vasodilators, neuroendocrine alterations $(9,10)$.

The presence and progression of inflammatory alterations of the oesophageal and duodenal mucous membranes in the patient cohort concerned appear to contribute to formation of a particular AP profile (11). Close pathogenetic interrelations in the form of systemic impairments at the level of arterioles, neuroendocrine shifts, and changes in haemostasis have been confirmed (12). Studies of other authors demonstrated that haemodynamic disorders characteristic of AH play a significant role in the pathogenesis of acid-dependent diseases (13-15).

A comorbid condition of patients, as a rule, requires prescription of several drugs. The findings of our study showed that the most frequently prescribed combination of drugs in patients with AH and ADD was that of omeprazole and amlodipine.

The key reaction of phase I metabolism of omeprazole is formation of 5-hydroxiomeprazole, catalyzed by CYP2D19 and CYP3A4. Affinity of omeprazole to $3 \mathrm{~A} 4$ is 10 times lower than that to $2 \mathrm{C} 19$, therefore, metabolism via $3 \mathrm{~A} 4$ is considered as an alternative pathway while creating high concentrations of omeprazole in blood plasma of patients (1619).

The known fact is that most drugs used for treatment of cardiovascular diseases are metabolized by isoenzyme of cytochrome P450 3A4, including also amlodipine (20). In this connection, we studied possible influence of omeprazole on antihypertensive efficacy of amlodipine in patients with AH and ADD.

Characteristic of amlodipine is a direct correlation between the degree of the antihypertensive effect and plasma concentration. One of the factors determining the concentration of the drug is metabolism intensity which, in turn, depends on the activity of amlodipine-metabolizing isoenzyme cytochrome CYP3A4 $(21,22)$.

Omeprazole has a dual-pathway metabolism: via CYP 2C19 and CYP 3A4. In case of the presence of a particular genetic polymorphism of isoenzyme of cytochrome CYP 2C19 P450, metabolism of omeprazole may be shifted towards CYP 3A4 P450, which, probably, may lead to a decrease in the activity of CYP 3A4, slowing-down of amlodipine metabolism and, as a consequence, to an increase in its concentration in blood plasma (23-25). Elevated blood plasma amlodipine leads to enhancement of the antihypertensive effect, which we observed in patients who on the background of antihypertensive therapy with amlodipine were taking pharmacotherapy with omeprazole due to exacerbation of aciddependent diseases. Patients undergoing therapy with amlodipine supplemented with omeprazole were found to have a statistically significant decrease in average circadian SAP, average circadian DAP, mean value of daytime SAP, mean values of nighttime SAP $(p<0.04)$ as compared with patients not taking therapy with omeprazole.

Simultaneous administration of amlodipine and omeprazole in patients with AH and ADD turned out to enhance the antihypertensive effect of amlodipine, which probably is the result of substrate competition of amlodipine and omeprazole at the level of isoenzyme of cytochrome CYP 3A4 P450.

\section{REFERENCES}

1. Khlynova OV, Tyev AV, Berseneva LN. Problem of comorbidity with regard for the state of the cardiovascular system in patients with arterial hypertension and acid-dependent diseases. Kazan Medical Journal.2003; 1: 80-6.

2. Vertkin AL, Skotnikov AS. Comorbidity. Attending Physician. 2013;6:6-9.

3. Ovchinnikova EA. Role of monitoring of safety of drugs in solving the problem of their rational use. Good Clinical Practice. 2003;4:88-95.

4. Sizova ZhM, Shikh EV., Baichorov IKh. Polyprogmasia and drug interaction in treatment of comorbid patients with arterial hypertension an ambulatory-polyclinical practice. Polyclinic. Cardiology. 2015;1:13-8.

5. Clinical Pharmacology: manual. Under the editorship of V. G. Kukes and D. A. Sychev. Moscow: GEOTAR-Media; 2015.

6. Kukes VG, Grachev SV, Sychev SV, Ramenskaya GV. Metabolism of Drugs. Scientific Grounds of Personified Medicine. Moscow: GEOTAR-Media; 2008. 
7. Andersson TB, Ahlström M. Comparison of inhibitory effects of the proton pump-inhibiting drugs omeprazole, esomeprazole, lansoprazole, pantoprazole, and rabeprazole on human cytochrome P450 activities. Drug Metab Dispos.2004 Aug;32(8):821-27. https://doi.org/10.1124/dmd.32.8.821 PMid:15258107

8. Howden CW. Clinical pharmacology of omeprazole. Clin Pharmacokinet.1991;20:38-49. https://doi.org/10.2165/00003088-199120010-00003 PMid:2029801

9. Leushina EA. Chicherina EN. Arterial hypertension and gastric motor-evacuatory disorders. Vyatka Medical Review.2012;3:50-65.

10. Butov MA. On aetiology and pathogenesis of peptic ulcer. Experimental and Clinical Gastroenterology. 2003;6:59.

11. Kokarovtseva LV, Tuev AV, Khlynova OV. Structural and functional remodelling of the heart in patients with combination of hypertension and gastroesophageal reflux disease. Perm Medical J. 2008;5(25):65-70.

12. Samsonov AA, Kazyulin AN, Salman IK, Lebedeva EG. Role of vascular pathology in pathogenesis of peptic ulcer disease. Archives of Internal Medicine.1992;64(2):138-141.

13. Krylov AA. On the problem of compatibility of diseases. Clin. Med. 2000;1:56-68.

14. Smirnova LE. On the problem of comorbidity of ulcerative-erosive gastroduodenal lesions and arterial hypertension. Clin. Med. 2003;3:9-15.

15. Smirnova LE, Shpak LV, Vinogradov VF. Peculiarities of a comorbid course of ulcerative-erosive gastroduodenal lesions and arterial hypertension. Clin. Med. 2005;4:43-54.

16. Leonova MV. Genetic polymorphism of CYP2C19 - a predictor of clinical efficacy of proton pump inhibitors. General Medicine. Clinical Pharmacology. 2015;4:30-39.

17. Lopina OD, Serebrova SYu. Main pharmacokinetic characteristics of proton pump inhibitors and efficacy of their action. Manual for physicians; 2016.

18. Kuo $\mathrm{CH}$, Lu CY, Shih HY. CYP2C19 polymorphism influences Helicobacter pylori eradication. World J. Gastroenterol. 2014;20(43):16029-16036. https://doi.org/10.3748/wjg.v20.i43.16029 PMid:25473155 PMCid:PMC4239489

19. Li WW, Wallhagen MI, Froelicher ES. Hypertension control, predictors for medication adherence and gender differences in older Chinese immigrants. J Adv Nurs. 2008 Feb;61(3):326-335. https://doi.org/10.1111/j.13652648.2007.04537.x PMid:18197867

20. Morozova TV, Sychev DA, Shikh NV. Pharmacogenetic approaches to predicting efficacy and safety of amlodipine in hypertensive patients. Attending Physician. 2016;3:77-82.

21. Morozava TE, Andrushchishina TB. Modern aspects of pharmacotherapy of arterial hypertension: possibilities of amlodipine. Attending Physician. 2013;2:7-12.

22. Leonova MV. Clinical pharmacology and clinical efficacy of amlodipine. Data of evidence-based medicine. Medical Council. 2014;12:14-21.

23. Wedemeyer RS, Blume H. Pharmacokinetic drug interaction profiles of proton pump inhibitors: an update. Drug Saf.2014, Apr;37(4):201-211. https://doi.org/10.1007/s40264-014-0144-0 PMid:24550106 PMCid:PMC3975086

24. Shikh EV, Sychev DA. Pantoprazole - safety in drug-drug interaction. Russian Journal of Gastroenterology, Hepatology and Coloproctology. 2012;22(5):4-12.

25. Cheng JW, Frishman WH, Aronow WS. Updates on Cytochrome P450-Mediated Cardiovascular Drug Interactions. Dis Mon. 2010 Mar;56(3):163-179. https://doi.org/10.1016/j.disamonth.2009.12.011 PMid:20189501

$\diamond \diamond \diamond \diamond \diamond \diamond \diamond$

http://www.ejgm.co.uk 